Pacific Journal of Mathematic 


\title{
EXTREME OPERATORS ON CHOQUET SIMPLEXES
}

\author{
KA-SING LAU
}

\begin{abstract}
If $K$ is a Choquet simplex and $X$ is a metrizable compact Hausdorff space, we let $\partial_{S} K$ denote the set of extreme points of $K$ with the facial topology and let $S(L(C(X), A(K)))$ denote the set of continuous operators from $C(X)$ into $A(K)$ with norm not greater than 1. Our main purpose in this paper is to characterize the extreme points of $S(L(C(X), A(K)))$. We show that $T$ is an extreme point of $S(L(C(X), A(K)))$ if and only if its adjoint $T^{*}$ sends extreme points of $K$ into $X \cup-X \subseteq C(X)^{*}$, also, the set of extreme points of $S(L(C(X), A(K)))$ equals $C\left(\partial_{S} K, X \cup-X\right)$.
\end{abstract}

1. Suppose $E_{1}, E_{2}$ are two real Banach spaces, we let $S\left(E_{1}\right)$ denote the unit ball of $E_{1}$ and let $L\left(E_{1}, E_{2}\right)$ be the set of continuous linear operators from $E_{1}$ into $E_{2}$. Following Morris and Phelps [7], we call an operator $T$ in $S\left(L\left(E_{1}, E_{2}\right)\right.$ ) a nice operator if its adjoint $T^{*}$ sends extreme points of $S\left(E_{1}^{*}\right)$ into extreme points of $S\left(E_{2}^{*}\right)$. It is clear that if $T$ is a nice operator, then $T$ is an extreme point of $S\left(L\left(E_{1}, E_{2}\right)\right)$. The converse is in general not true and there are various literatures dealing with this problem under different hypotheses (c.f. [2], [5], [9]). In [2], Blumenthal, Lindenstrauss, Phelps proved the following: Suppose $E_{1}=C(X), E_{2}=C(Y)$ where $X, Y$ are compact Hausdorff spaces with $X$ metrizable, then $T$ is an extreme point of $S(L(C(X), C(Y)))$ if and only if there exists a continuous $\operatorname{map} \varphi: Y \rightarrow X$ and a continuous function $\lambda \in C(X),|\lambda|=$ 1 such that $(T f)(y)=\lambda(y) f(\varphi(y))$ for $y \in Y$ and $f \in C(X)$. As a simple consequence, we see that in such case, $T$ is a nice operator. Our main purpose in this paper is to prove a similar characterization for an extreme point $T \in S(L(C(X), A(K)))$ where $X$ is a metrizable compact Hausdorff space and $A(K)$ is the set of continuous affine functions on a Choquet simplex $K$.

Suppose $K$ is a Choquet simplex, we let $\partial K$ be the set of extreme points of $K$ and let $\partial_{S} K$ be the set $\partial K$ with the facial topology. A necessary and sufficient condition for a real-valued function $f$ on $\partial_{S} K$ to be continuous is that for any $a \in A(K)$, there exists $b \in A(K)$ such that $b(x)=f(x) \cdot a(x), x \in \partial K$ (c.f. [1]). In $\S 2$, we extend this property to an $E$-valued function where $E$ is a Fréchet space and we will make use of the results in the next two sections. In $\S 3$, we prove our main theorem: let $K$ be a Choquet simplex and $X$ a metrizable compact Hausdorff space, then $T \in S(L(C(X), A(K)))$ is an extreme point if and only if there exists $\varphi \in A\left(K, C(X)^{*}\right)$ (set of affine $w^{*}$ continuous functions from $K$ into $\left.C(X)^{*}\right)$ and $a \lambda \in A(K)$ such that 
(i) $\varphi(\partial K) \subseteq X$; (ii) $|\lambda(x)|=1$ for $x \in \partial K$; (iii) $T f(x)=\lambda(x) \cdot \varphi(x)(f), f \in$ $C(K), x \in \partial K$. From this, we can easily see that such $T$ is a nice operator. Analogous results have also been obtained by Lazar [4] in considering the compact operators and positive operators from $C(X)$ into $A(K)$. In $\S 4$, we prove that the set of extreme points of $S(L(C(X), A(K)))$ equals the set $C\left(\partial_{S} K, X \cup-X\right)$ where we consider $X$ and $-X$ to be in $C(X)^{*}$. By using this result, we can show that $S(L(C(X), A(K)))$ is the weak operator closed convex hull of its extreme points if and only if $\partial_{S} K$ is totally disconnected. In such case, $K$ is a Bauer simplex and $A(K)=C(Y)$ for some compact Hausdorff space $Y$. The above is a generalization of a result by Morris and Phelps [7] on considering $S(L(C(X), C(Y)))$.

2. Suppose $K$ is a simplex (Choquet), we let $\partial K$ be the set of extreme points of $K$ and let $\partial_{S} K$ be the set $\partial K$ with the facial topology. Recall that a set $A$ is facially closed if and only if $A=\varnothing$ or $A=$ $F \cap \partial K$ where $F$ is a closed face of $K$. It is known that [1] $\partial_{S} K$ is compact (not necessarily Hausdorff) and that every real-valued continuous function on $\partial_{S} K$ can be extended to a continuous affine function on $K$. Our first theorem is to generalize this result to an $E$-valued function where $E$ is a Fréchet space. We will use $A(K, E)$ to denote the set of continuous affine functions from $K$ into $E$.

Lemma 2.1. Let $K$ be a simplex and let $E$ be a locally convex space. Suppose $f$ is a continuous function from $\partial_{S} K$ to $E$ and $U i s$ an open convex symmetric neighborhood of 0 in $E$, then there exists a continuous affine function $T$ in $A(K, E)$ such that $T(x)-f(x) \in U$ for each $x \in \partial K$. Moreover, $T$ can be chosen such that $T / \partial K$ is facially continuous.

Proof. By the above remark, we see that the lemma is true for $E=R$. If $E=R^{n}$, we can write $f=\left(f_{1}, \cdots, f_{n}\right)$ where each $f_{i}$ : $\partial_{S} K \rightarrow R, 1 \leqq i \leqq n$, is continuous and hence there exist extensions $T_{i}$ of $f_{i}$ on $K, 1 \leqq i \leqq n$. Letting $T=\left(T_{1}, \cdots, T_{n}\right)$ shows that the lemma is true for $E=R^{n}$.

We now pass to prove the general case. Since $\partial_{S} K$ is a compact set, $f\left(\partial_{S} K\right)$ is compact. Hence there exist $x_{1}, \cdots, x_{n}$ in $\partial_{S} K$ such that $\left\{f\left(x_{i}\right)+U\right\}_{i=1}^{n}$ is an open cover of $f\left(\partial_{S} K\right)$. Let $\left\{\alpha_{1}, \cdots, \alpha_{n}\right\}$ be a partition of unity subordinated to $\left\{f\left(x_{i}\right)+U\right\}_{i=1}^{n}$ i.e., each $\alpha_{i}, i=1, \cdots, n$ is real-valued, nonnegative continuous function on $\partial_{S} K$ such that $\operatorname{supp} \alpha_{i} \subseteq f\left(x_{i}\right)+U, \sum_{i=1}^{n} \alpha_{i}=1$. Define $f_{U}: \partial_{S} K \rightarrow E$ by

$$
f_{U}(x)=\sum_{i=1}^{n} \alpha_{\imath}(f(x)) f\left(x_{i}\right) \text {. }
$$


Then $f_{U}$ is continuous and for $x \in \partial_{S} K$

$$
f_{U}(x)-f(x)=\sum_{i=1}^{n} \alpha_{\imath}(f(x))\left(f\left(x_{i}\right)-f(x)\right) \in U .
$$

Also, notice that $f_{U}\left(\partial_{S} K\right)$ is contained in a finite-dimensional subspace of $E$, so we can extend $f_{U}$ to $T_{U} \in A(K, E)$ and

$$
T_{U}(x)-f(x)=f_{U}(x)-f(x) \in U, x \in \partial K .
$$

THEOREM 2.2. Suppose $K$ is a simplex and $E$ is a Fréchet space; then every continuous function from $\partial_{S} K$ into $E$ can be extended to a continuous affine function in $A(K, E)$.

Proof. Let $\left\{p_{n}\right\}$ be an increasing sequence of pseudo norms on $E$ which defines the topology of $E$ and let $B_{n}(r)=\left\{y \in E: p_{n}(y)<r\right\}$. We construct a sequence $\left\{T_{n}\right\}$ in $A(K, E)$ as follows: Let $T_{1} \in A(K$, $E)$ be such that

$$
T_{1}(x) \in f(x)+B_{1}\left(2^{-1}\right), x \in \partial K \text {. }
$$

Suppose we have constructed $T_{n}$; let $T_{n+1} \in A(K, E)$ satisfy

$$
T_{n+1}(x) \in\left(f(x)-\sum_{k=1}^{n} T_{k}(x)\right)+B_{n}\left(2^{-n}\right), x \in \partial K .
$$

(Note that $\left(f(x)-\sum_{k=1}^{n} T_{k}(x)\right)$ is facially continuous on $\partial K$, hence we can apply Lemma 2.1.)

The function $\sum_{n=1}^{\infty} T_{n}$ converges uniformly to $f$ on $\partial K$. We want to show that $\sum_{n=1}^{\infty} T_{n}$ is uniformly Cauchy on $K$ and thus will converge to a continuous affine function $T \in A(K, X)$ such that $T(x)=f(x), x \in$ $\partial K$. Indeed, for any $p \in\left\{p_{n}\right\}$, the function $p \circ\left(\sum_{n=m}^{k} T_{n}\right): K \rightarrow R$ is continuous and convex, it attains maximum on some extreme point $x_{0} \in K$, hence

$$
p\left(\sum_{n=m}^{k} T_{n}(x)\right) \leqq p\left(\sum_{n=m}^{k} T_{n}\left(x_{0}\right)\right), x \in K .
$$

It follows that $\sum_{n=m}^{k} T_{n}(x)$ converges uniformly to 0 as $k, m$ tend to infinity. Thus $\sum_{n=1}^{\infty} T_{n}$ converges to $T \in A(K, E)$ and the proof is completed.

Let $K$ be a compact convex set and $E$ a locally convex space. We use $c(E)$ to denote the family of all nonempty convex subsets of $E$ and let $\bar{c}(E)$ be the subfamily of $c(E)$ consisting of all nonempty closed convex subsets of $E$. A set-valued map $\Phi$ from $K$ into $c(E)$ is convex if 


$$
\lambda \Phi\left(x_{1}\right)+(1-\lambda) \Phi\left(x_{2}\right) \leqq \Phi\left(\lambda x_{1}+(1-\lambda) x_{2}\right), 0 \leqq \lambda \leqq 1, x_{1}, x_{2} \in K .
$$

The map $\Phi$ is called lower semicontinuous if for each open set $U$ in $E$, the set $\{x \in K: \Phi(x) \cap U \neq \varnothing\}$ is an open set in $K$ and a function $\varphi: K \rightarrow E$ is called a selection of $\Phi$ if $\varphi(x) \in \Phi(x)$ for each $x \in K$.

Lemma 2.3. (Lazar [5]). Suppose $K$ is a simplex and $E$ is a Fréchet space. Let $\Phi: K \rightarrow \bar{c}(E)$ be a convex, lower semicontinuous map. Then $\Phi$ admits a continuous affine selection $\varphi$. Moreover, if $F$ is a closed face of $K$ and $f: F \rightarrow E$ is a continuous affine selection of $\Phi / F$, then the selection $\varphi$ can be chosen so that $\varphi / F=f$.

We remark that if we replace $E$ by a complete locally convex space and assume that for each $x \in K, \Phi(x)$ is contained in a bounded convex set which is metrizable with the relative topology, then the above lemma still holds.

THeORem 2.4. Let $K$ be a simplex and let $E$ be a Fréchet space. Suppose $f$ is a facially continuous real-valued function on $\partial K$. Then for any $T \in A(K, E)$. There exists $S \in A(K, E)$ such that

$$
S(x)=f(x) T(x), x \in \partial K \text {. }
$$

Proof. The theorem is true for $E=R[1]$. For $E=R^{n}$, we can write $T=\left(T_{1}, \cdots, T_{n}\right)$ and there exists $S_{i} \in A(K)$ such that

$$
S_{i}(x)=f(x) T_{i}(x), x \in \partial K, \quad i=1, \cdots, n .
$$

Hence the function $S=\left(S_{1}, \cdots, S_{n}\right) \in A\left(K, R^{n}\right)$ satisfies the requirement. To consider the general case, we let $U$ be an open convex neighborhood of 0 in $E$ and claim that there exists $S_{U} \in A(K, E)$ such that

$$
S_{U}(x) \in f(x) T(x)+\bar{U}, x \in \partial K .
$$

Since $f \in C\left(\partial_{S} K\right)$ and $\partial_{S} K$ is compact, $f$ has a bound $M$. The map $T$ is continuous. It follows that $T(K)$ is compact and there exists $x_{1}, \cdots, x_{n} \in K$ such that $\bigcup_{i=1}^{n}\left(T\left(x_{i}\right)+(1 / M) U\right)$ covers $T(K)$. Define $\Phi: K \rightarrow \bar{c}\left(R^{n}\right)$ by

$$
\begin{aligned}
\Phi(x) & =\left\{\left(\lambda_{i}\right) \in R^{n}: \sum_{i=1}^{n} \lambda_{i} T\left(x_{i}\right) \in T(x)+\frac{1}{M} \bar{U}, \sum_{i=1}^{n} \lambda_{i}\right. \\
& \left.=1, \lambda_{i} \geqq 0, i=1, \cdots, n\right\} .
\end{aligned}
$$

It is easy to show that $\Phi$ is lower semicontinuous and satisfies hypotheses of Lemma 2.3. Hence there is a continuous affine selection $\varphi$ 
of $\Phi$. By the first part of the proof, we can find $S_{U}^{\prime}: K \rightarrow R^{n}$ such that

$$
S_{U}^{\prime}(x)=f(x) \varphi(x), x \in \partial K
$$

Define $S_{U}: K \rightarrow E$ by

$$
S_{U}(x)=\sum_{i=1}^{n}\left(S_{U}^{\prime}\right)_{i}(x) T\left(x_{i}\right),
$$

where $S_{U}^{\prime}=\left(\left(S_{U}^{\prime}\right)_{1}, \cdots,\left(S_{U}^{\prime}\right)_{n}\right)$. We see that for $x \in \partial K$

$$
S_{U}(x)=\sum_{i=1}^{n} f(x) \varphi_{i}(x) T\left(x_{i}\right)=f(x) \sum_{i=1}^{n} \varphi_{i}(x) T\left(x_{i}\right) \in f(x) T(x)+\bar{U} .
$$

To complete the proof, we let $\left\{p_{n}\right\}$ be an increasing sequence of pseudo norms which defines the topology on $E$ and let $B_{n}(r)=\{y \in$ $\left.E: p_{n}(y) \leqq r\right\}$. By the above, we can find a sequence $S_{n} \in A(K, E)$ such that

$$
S_{n}(x) \in f(x) T(x)+B_{n}\left(2^{-n}\right), x \in \partial K .
$$

Similar to Theorem 2.2, we can show that the sequence converges uniformly on $\partial K$ and hence converges uniformly on $K$ to $S \in A(K, E)$ such that

$$
S(x)=f(x) T(x), x \in \partial K .
$$

We remark that above theorem will also hold if we replace $E$ by a complete locally convex space such that every bounded subset of $E$ is metrizable.

3. It is known that if $K$ is a simplex and $E$ is a Banach space, for $T \in L(E, A(K))$, there exists a $w^{*}$-continuous affine function $T^{*}$ : $K \rightarrow E^{*}$ such that $T(x)(k)=\left(T^{*}(k)\right)(x)$ for each $x \in E, k \in K$. Moreover, $\|T\|=\sup _{x \in K}\left\|T^{*}(k)\right\|$. Conversely, for each $w^{*}$-continuous affine function $T^{*}$ from $K$ into $E^{*}$, there corresponds an operator $T: E \rightarrow$ $A(K)$ satisfies the above two conditions. Hence, for convenience, we will identify $A\left(K, E^{*}\right)$, the space of $w^{*}$-continuous affine functions from $K$ into $E^{*}$, with $L(E, A(K))$. In this section, our main object is to prove the following theorem.

THeorem 3.1. Let $K$ be a simplex and let $X$ be a metrizable compact Hausdorff space. Then a continuous linear operator $T$ in $L(C(X), A(K))$ is an extreme point of $S(L(C(X), A(K)))$ if and only if there exists a map $\varphi \in A\left(K, C(X)^{*}\right)$ and a map $\lambda \in A(K)$ such that

( i ) $\varphi(\partial K) \leqq X \leqq C(X)^{*}$

(ii) $|\lambda(x)|=1, x \in \partial K$ 
(iii)

$$
T(f)(x)=\lambda(x) \cdot \varphi(x)(f), f \in C(X), x \in \partial K
$$

As an easy consequence we have:

Theorem 3.2. Suppose $K$ is a simplex and $X$ is a metrizable compact Hausdorff space. Then $T \in S(L(C(X), A(K)))$ is an extreme point if and only if $T$ is a nice operator. Equivalently, $T^{*}(\partial K) \subseteq$ $X \cup-X$, where $X$ and $-X$ are considered to be in $C(X)^{*}$.

Blumenthal-Lindenstrauss-Phelps [2] proved the above theorem for the case when $A(K)=C(Y)$ for some compact Hausdorff space $Y$. We employ a similar idea to prove this general case. In [4], Lazar considered the set of compact operators and set of positive operators of similar type. He also gave an example showing the above theorem is no more valid for the operator space $L(A(K), C(X))$. We will begin by listing and proving a series of lemmas which will lead to the theorem.

Lemma 3.3. Suppose $K$ is a topological space and $E$ is a locally convex space. Let $\Phi: K \rightarrow c(E)$ be a set valued map satisfying

(i) there exists a compact set $K^{\prime} \subseteq E$ such that $\Phi(x) \subseteq K^{\prime}$ for each $x \in K$,

(ii) for each open half space $H$ in $E$ (i.e., $H=\{y \in E: f(y)<r\}$, $\left.f \in E^{*}, r \in R\right)$, the set $\{x \in K: \Phi(x) \cap H \neq \varnothing\}$ is open in $K$.

Then $\Phi$ is lower semicontinuous.

Proof. C.f. [3].

Lemma 3.4. Let $K$ be a simplex and let $X$ be a metrizable compact Hausdorff space. Let $T \in A\left(K, C(X)^{*}\right),\|T\| \leqq 1$. Define $\Phi: K \rightarrow$ $\bar{c}\left(C(X)^{*}\right)$ by

$$
\begin{gathered}
\Phi(x)=\operatorname{cl}\left\{\mu: 0 \leqq \mu \leqq \sum_{i=1}^{n} \lambda_{i} T_{i}\left(k_{i}\right)^{+}, \sum_{i=1}^{n} \lambda_{i} k_{i}=x, \sum_{i=1}^{n} \lambda_{i}=1, \lambda_{i} \geqq 0,\right. \\
\left.k_{i} \in K, i=1, \cdots, n\right\},
\end{gathered}
$$

where the closure is taken under the $w^{*}$-topology. Then we have

(i) $\Phi(\lambda x+(1-\lambda) y)=\lambda \Phi(x)+(1-\lambda) \Phi(y) ; 0<\lambda<1, x, y \in K$.

(ii) $\Phi$ is lower semicontinuous.

Proof. Note that for any open set $U$ and any subset $A$ in a topological space, $U \cap A \neq \varnothing$ if and only if $U \cap \bar{A} \neq \varnothing$; hence it suffices to prove the lemma by showing that 


$$
\begin{aligned}
\Psi(x) & =\left\{\mu: 0 \leqq \mu \leqq \sum_{i=1}^{n} \lambda_{i} T_{i}\left(k_{i}\right)^{+}, \sum_{i=1}^{n} \lambda_{i} k_{i}=x, \sum_{i=1}^{n} \lambda_{i}\right. \\
& \left.=1, \lambda_{i} \geqq 0, k_{i} \in K, i=1, \cdots, n\right\}
\end{aligned}
$$

satisfies (i) and (ii).

(i) It is clear that $\Psi(\lambda x+(1-\lambda) y) \supseteqq \lambda \Psi(x)+(1-\lambda) \Psi(y)$. To prove the reverse inclusion, let $0 \leqq \mu \leqq \sum_{i=1}^{n} \lambda_{i} T\left(k_{i}\right)^{+}$, where $\sum_{i=1}^{n} \lambda_{i} k_{i}=$ $\lambda x+(1-\lambda) y, \sum_{i=1}^{n} \lambda_{i}=1, \lambda_{i} \geqq 0, k_{i} \in K, i=1, \cdots, n$. By the decomposition lemma [8] on lattices, there exist $\left\{\lambda_{i j}\right\},\left\{k_{i j}\right\}$ such that $\lambda_{i j}>$ $0, k_{i j} \in K, i=1, \cdots, n, j=1,2$ and

$$
\begin{aligned}
& \lambda x=\sum_{i=1}^{n} \lambda_{i 1} k_{i 1},(1-\lambda) y=\sum_{i=1}^{n} \lambda_{i 2} k_{i 2}, \\
& \lambda_{i} k_{i}=\lambda_{i 1} k_{i 1}+\lambda_{i 2} k_{i 2}, \quad i=1, \cdots, n \text {. }
\end{aligned}
$$

Hence

$$
0 \leqq \mu \leqq \lambda\left(\sum_{i=1}^{n} \frac{\lambda_{i 1}}{\lambda} T\left(k_{i 1}\right)^{+}\right)+(1-\lambda)\left(\sum_{i=1}^{n} \frac{\lambda_{i 2}}{1-\lambda} T\left(k_{i 2}\right)^{+}\right) .
$$

The Riesz decomposition property implies that there exist $\mu_{1}, \mu_{2} \in$ $C(X)^{*}$ such that

$$
\begin{aligned}
& 0 \leqq \lambda \mu_{1} \leqq \lambda \sum_{i=1}^{n} \frac{\lambda_{i 1}}{\lambda} T\left(k_{i 1}\right)^{+} \\
& 0 \leqq(1-\lambda) \mu_{2} \leqq(1-\lambda) \sum_{i=1}^{n} \frac{\lambda_{i 2}}{\lambda} T\left(k_{i 2}\right)^{+}
\end{aligned}
$$

and

$$
\mu=\lambda \mu_{1}+(1-\lambda) \mu_{2} .
$$

Hence we have

$$
\Psi(\lambda x+(1-\lambda) y) \leqq \lambda \Psi(x)+(1-\lambda) \Psi(y) .
$$

(ii) Let $V(\lambda, \mu, f)=\left\{\nu: \nu(f)-\mu(f)<\lambda^{-1}\right\}, f \in C(X), \mu \in C(X)^{*}, \lambda>$ 0 . By Lemma 3.3, we need only show that the set $\{x \in K: \Psi(x) \cap V(1$, $\mu, f) \neq \varnothing\}$ is open in $K$, or equivalently, we will prove the following:

(*) let $x \in K, \mu \in \Psi(x)$, then there exists a neighborhood $U$ of $x$ such that $V(1, \mu, f) \cap \Psi(k) \neq \varnothing$ for each $k \in U$.

Since $0 \leqq \mu \leqq \sum_{i=1}^{n} \lambda_{i} T\left(k_{i}\right)^{+}$, by the Reisz decomposition property, we can write $\mu=\sum_{i=1}^{n} \lambda_{i} \mu_{i}$ where $0 \leqq \mu_{i} \leqq T\left(k_{i}\right)^{+}, i=1, \cdots, n$. We first observe that if one of the $i$, say $i=1$, has a neighborhood $U_{1}$ of $k_{1}$ such that for $k \in U_{1}$ 


$$
V\left(\lambda_{1}, \mu_{1}, f\right) \cap \Psi(k) \neq \varnothing,
$$

then $\left(^{*}\right)$ will hold, for the set $\lambda_{1} U_{1}+\sum_{i=2}^{n} \lambda_{i} k_{i}$ is the neighborhood we need. Now suppose $\left({ }^{*}\right)$ does not hold. By the above remark, there exist nets $\left\{k_{i}^{\alpha}\right\}, i=1, \cdots, n$ such that $\left\{k_{i}^{\alpha}\right\}$ converges to $k_{i}$ and

$$
V\left(\lambda_{i}, \mu_{i}, f\right) \cap \Psi\left(k_{i}^{\alpha}\right)=\varnothing \text { for each } k_{i}^{\alpha} .
$$

Consider the net $\left\{\sum_{i=1}^{n} \lambda_{i} k_{i}^{\alpha}\right\}$; it converges to $x$ and

$$
V(1, \mu, f) \cap \Psi\left(\sum_{i=1}^{n} \lambda_{i} k_{i}^{\alpha}\right)=\varnothing \text { for each } \alpha .
$$

(The proof of this is straightforward, by using (i).) Since $\left\{T\left(k_{i}^{\alpha}\right)^{+}\right\}_{\alpha}$ is contained in a compact set (for $\|T\| \leqq 1$ ), we may assume that $T\left(k_{i}^{\alpha}\right)^{+}$converges to $\nu_{i}$ and $T\left(k_{i}^{\alpha}\right)^{-}$converges to $\nu_{i}^{\prime}, i=1, \cdots, n$. Thus

$$
T(x)=\sum_{i=1}^{n} \lambda_{i} T\left(k_{i}\right)^{+}-\sum_{i=1}^{n} \lambda_{i} T\left(k_{i}\right)^{-}=\sum_{i=1}^{n} \lambda_{i} \nu_{i}-\sum_{i=1}^{n} \lambda_{i} \nu_{i}^{\prime} .
$$

It is clear that $\nu_{i} \geqq T\left(k_{i}\right)^{+}$. Let $\omega=\sum_{i=1}^{n} \lambda_{i} \nu_{i}$; since $\sum_{i=1}^{n} \lambda_{i} T\left(k_{i}\right)^{+} \geqq$ $\mu$, we have $\omega \geqq \mu$. By Radon Nikodym's theorem, we can write $d \mu=$ $g_{1} d \omega$ where $g_{1}$ is a Borel function on $K$ such that $0 \leqq g_{1} \leqq 1$. Let $g \in C(X)$ be such that

$$
\int_{X}\left|g-g_{1}\right| d \omega \leqq(2\|f\|)^{-1} .
$$

If we define $\mu_{\alpha}$ by $d \mu_{\alpha}=g \cdot d\left(\sum_{i=1}^{n} \lambda_{i} T\left(k_{i}^{\alpha}\right)^{+}\right)$, then $\mu_{\alpha} \in \Psi\left(\sum_{i=1}^{n} \lambda_{i} k_{i}^{\alpha}\right)$ for each $\alpha$ and

$$
\mu_{\alpha}(f)=\int_{X} f d \mu_{\alpha}=\int_{X} f g d\left(\sum_{i=1}^{n} \lambda_{i} T\left(k_{i}^{\alpha}\right)^{+}\right) \longrightarrow \int_{X} f g d \omega .
$$

Hence

$$
\lim \sup \left(\mu_{\alpha}(f)-\mu(f)\right) \leqq \int_{X}\left|f g-f g_{1}\right| d \omega \leqq \frac{1}{2},
$$

so that $\left\{\mu_{\alpha}\right\}$ is eventually in $V(1, \mu, f) \cap \Psi\left(\sum_{i=1}^{n} \lambda_{i} k_{i}^{\alpha}\right)$. This contradicts $\left({ }^{*}\right)$ and we conclude that $\left(^{*}\right)$ is true.

LEMma 3.5. Let $K$ be a simplex and let $X$ be a metrizable compact Hausdorff space. Suppose there are set-valued maps $\Phi, \Psi: K \rightarrow$ $\bar{c}\left(C(X)^{*}\right)$ satisfies $\log y$ ).

(i) $\Phi, \Psi$ are convex, lower semicontinuous (w.r.t. the $w^{*}$-topo-

(ii) There exists a bounded set $W$ contained in the positive cone of $C(X)^{*}$ such that for each $x \in K, \Phi(x), \Psi(x) \subseteq W$.

(iii) $0 \in \Phi(x), \Psi(x)$ for each $x \in K$. 
Also, let $x_{0} \in \partial K, \mu_{0} \in \Phi\left(x_{0}\right), \nu_{0} \in \Psi\left(x_{0}\right)$ with $\left\|\mu_{0}\right\|=\left\|\nu_{0}\right\|$. Then there exist $T_{1}, T_{2} \in A\left(K, C(X)^{*}\right)$ selections of $\Phi, \Psi$ respectively such that $T_{1}\left(x_{0}\right)=$ $\mu_{0}, T_{2}\left(x_{0}\right)=\nu_{0}$ and $\left\|T_{1}(x)\right\|=\left\|T_{2}(x)\right\|$ for each $x \in K$.

Proof. Let us first consider the case $C(X)^{*}=R$. The map $x \rightarrow$ $\Phi(x) \cap \Psi(x)$ is well-defined (i.e., $\Phi(x) \cap \Psi(x) \neq \varnothing$ for all $x \in K$ ) and is lower semicontinuous, convex. Hence there exists a selection $T$ of the above map such that

$$
T\left(x_{0}\right)=\mu_{0}=\nu_{0} .
$$

(Note that in this case, $\mu_{0}=\nu_{0}$.) The maps $T_{1}, T_{2}=T$ meet the requirement.

To prove the general case, we let $N=\left\{\mu \in C(X)^{*}: \mu(1)=0\right\}$, and let $R \mu_{0}, R \nu_{0}$ be the subspaces generated by $\mu_{0}$ and $\nu_{0}$. (We assume that $\mu_{0}, \nu_{0} \neq 0$, otherwise the lemma is trivially true.) Then

$$
C(X)^{*}=N \oplus R \mu_{0}=N \oplus R \nu_{0} .
$$

Let $p_{1}, p_{2}$ be the projections of $C(X)^{*}$ onto $R \mu_{0}$ and $R \nu_{0}$. Define $\Phi_{0}: K \rightarrow \bar{c}\left(R \mu_{0}\right), \Psi_{0}: K \rightarrow \bar{c}\left(R \nu_{0}\right)$ as

$$
\begin{aligned}
& \Phi_{0}(x)=\left\{p_{1}(\mu): \mu \in \Psi(x)\right\}, \quad x \in K, \\
& \Psi_{0}(x)=\left\{p_{2}(\mu): \mu \in \Phi(x)\right\}, \quad x \in K \text {. }
\end{aligned}
$$

It is clear that we may apply the above remark and find $f_{1} \in A(K$, $\left.R \mu_{0}\right), f_{2} \in A\left(K, R \nu_{0}\right)$ such that

$$
f_{1}\left(x_{0}\right)=\mu_{0}, f_{2}\left(x_{0}\right)=\nu_{0},\left\|f_{1}(x)\right\|=\left\|f_{2}(x)\right\|, \quad x \in K .
$$

Since every bounded set in $C(X)^{*}$ is metrizable under the $w^{*}$-topology, we let $\left\{q_{n}\right\}$ be an increasing sequence of pseudo norms on $C(X)^{*}$ which defines the relative $w^{*}$-topology on $W$ and let $B_{n}(r)=\left\{\mu \in C(X)^{*}\right.$ : $\left.q_{n}(\mu) \leqq r\right\}$. We will construct two sequences $\left\{T_{1}^{n}\right\}$ and $\left\{T_{2}^{n}\right\}$ as follows: Let

$$
\begin{array}{ll}
\Phi_{1}(x)=\left(p_{1}^{-1}\left(f_{1}(x)\right)+B_{1}\left(2^{-1}\right)\right) \cap \Phi(x), & x \in K, \\
\Psi_{1}(x)=\left(p_{2}^{-1}\left(f_{2}(x)\right)+B_{1}\left(2^{-1}\right)\right) \cap \Psi(x), & x \in K .
\end{array}
$$

The maps $\Phi_{1}, \Psi_{1}$ are lower semicontinuous. By Lazar's selection theorem (also by the remark of Lemma 2.3), there exist continuous affine selections $T_{1}^{1}, T_{2}^{1}$ of $\Psi_{1}, \Phi_{1}$ such that

$$
T_{1}^{1}\left(x_{0}\right)=\mu_{0}, T_{2}^{1}\left(x_{0}\right)=\nu_{0} .
$$

For $n>1$, we let for $x \subset K$, 


$$
\begin{aligned}
\Phi_{n}(x)= & \left(p_{1}^{-1}\left(f_{1}(x)\right)+B_{n}\left(2^{-n}\right)\right) \cap\left(T_{1}^{n-1}(x)\right. \\
& \left.+B_{n-1}\left(2^{-n+1}\right)\right) \cap\left(\Phi(x)+B_{n-1}\left(2^{-n+1}\right)\right), \\
\Psi_{n}(x)= & \left(p_{2}^{-1}\left(f_{2}(x)\right)+B_{n}\left(2^{-n}\right)\right) \cap\left(T_{1}^{n-1}(x)\right. \\
& \left.+B_{n-1}\left(2^{-n+1}\right)\right) \cap\left(\Psi(x)+B_{n-1}\left(2^{-n+1}\right)\right)
\end{aligned}
$$

and let $T_{1}^{n}, T_{2}^{n}$ be continuous affine selections of $\Phi_{n}, \Psi_{n}$ respectively such that

$$
T_{1}^{n}\left(x_{0}\right)=\mu_{0}, T_{2}^{n}\left(x_{0}\right)=\nu_{0} .
$$

It is clear that $\left\{T_{i}^{n}\right\}, i=1,2$ are uniformly Cauchy sequences and converge to $T_{1}, T_{2} \in A\left(K, C(X)^{*}\right)$ respectively. Moreover,

$$
T(x) \in p_{1}^{-1}\left(f_{1}(x)\right) \cap \Phi(x), T_{2}(x) \in p_{2}^{-1}\left(f_{2}(x)\right) \cap \Psi(x), \quad x \in K .
$$

Observe that the subspace $N$ has the property that each point in the intersection of a translation of $N$ with the positive cone has the same norm. Thus for $x \in K$ each point in $P_{1}^{-1}\left(f_{1}(x)\right) \cap \Phi(x), P_{2}\left(f_{2}(x)\right) \cap \Psi(x)$ $(i=1,2)$ has the same norm and it follows that

$$
\left\|T_{1}(x)\right\|=\left\|f_{1}(x)\right\|=\left\|f_{2}(x)\right\|=\left\|T_{2}(x)\right\| \quad x \in K .
$$

Lemma 3.6. (Lazar [4]). Let $K$ be a simplex, $X$ a metrizable compact Hausdorff space and let $S$ be the set of positive operators $T$ from $C(X)$ to $A(K)$ which satisfies $T(1)=1$. Then $T$ is an extreme point of $S$ if and only if there is a function $\varphi: K \rightarrow C(X)^{*}$ which is affine and $w^{*}$-continuous such that

$$
T(f)(x)=\varphi(x)(f), f \in C(X), x \in K
$$

and

$$
T^{*}(\partial K) \subseteq X
$$

To prove Theorem 3.1, we use the standard fact that $T$ is an extreme point of $S\left(A\left(K, C(X)^{*}\right)\right)$ if and only if $Q=0$ is the only $Q \in A\left(K, C(X)^{*}\right)$ for which $\|T(x) \pm Q(x)\| \leqq 1, x \in \partial K$.

Proof of Theorem 3.1. Let $T \in A\left(K, C(X)^{*}\right)=L(C(X), A(K))$. Consider the following cases:

Case I. For each $x \in \partial K$, either $T(x) \geqq 0$ or $T(x) \leqq 0$. In this case, $\|T(x)\|=|T(x)(1)|$. Hence the map $x \rightarrow\|T(x)\|$ is continuous and we claim that $1-\|T(x)\|=0$ for $x \in \partial K$. Suppose this is not true; then there exists an extreme point $x_{0}$ of $K$ such that $1-\left\|T\left(x_{0}\right)\right\|>$ 0 . The map $x \rightarrow 1-\left\|T\left(x_{0}\right)\right\|$ is a continuous concave function and by Edwards' extension theorem [1], there exists $h \in A(K)$ such that 


$$
1-\|T(x)\| \geqq h(x) \geqq 0, x \in K
$$

and

$$
h\left(x_{0}\right)=\frac{1-T\left(x_{0}\right)}{2} .
$$

Choose $\mu \in C(X)^{*}$ such that $\mu \neq 0,\|\mu\| \leqq 1$; then the map $x \rightarrow h(x) \mu$ is in $A\left(K, C(X)^{*}\right)$ and

$$
\|T(x) \pm h(x) \mu\| \leqq 1, x \in K,
$$

with $h(x) \mu \neq 0$, which contradicts the fact that $T$ is an extreme point of $S\left(A\left(K, C(X)^{*}\right)\right)$. We conclude that $\|T(x)\|=1$ for $x \in \partial K$.

Let $\tau: K \rightarrow R$ be the affine continuous function defined by $\tau(x)=$ $T(x)(1)$; then $|\tau(x)|=\|T(x)\|=1$ for $x \in \partial K$. Let $\lambda$ be the restriction of $\tau$ to $\partial K$. We claim that $\lambda$ is continuous in the facial topology on $\partial K$. In fact, $\lambda(x)= \pm 1$ at each $x$ in $\partial K$ and

$$
\lambda^{-1}(1)=\tau^{-1}(1) \cap \partial K .
$$

Since $\tau^{-1}(1)$ is a closed face in $K$, the set $\lambda^{-1}(1)$ is closed in the facial toplogy and the same is true for $\lambda^{-1}(-1)$. We conclude that $\lambda \epsilon$ $C\left(\partial_{S} K\right)$ and can be extended to a function in $A(K)$ [1]. By Theorem 2.4, there exists $T^{\prime} \in A\left(K, C(X)^{*}\right)$ such that

$$
T^{\prime}(x)=\lambda(x) T(x), x \in \partial K .
$$

Note that $T^{\prime} \geqq 0$ on $\partial K$; hence $T^{\prime} \geqq 0$ on $K$ and $T^{\prime}(x)(1)=1, x \in \partial K$. Let $F: C(X) \rightarrow A(K)$ be defined as $F(f)(x)=T^{\prime}(x)(f)$. By the above remark, we see that $F(1)=1$ and $F \geqq 0$. Hence Lemma 3.6 implies there exists a $w^{*}$-continuous affine map $\varphi: K \rightarrow C(X)^{*}$ such that $F(f)=$ $f \circ \varphi$ and $\varphi(K) \leqq X$. Thus we have

$$
T(x)(f)=\lambda(x)^{-1} T^{\prime}(x)(f)=\lambda(x) F(f)(x)=\lambda(x) \varphi(x)(f), \quad x \in \partial K .
$$

(Note that $\lambda(x)=1$ or -1 , for $x \in \partial K$; hence $\lambda(x)=\lambda(x)^{-1}$.)

Case II. There exists $x_{0} \in \partial K$ such that $T\left(x_{0}\right)=T\left(x_{0}\right)^{+}-T\left(x_{0}\right)^{-}$, where the decomposition is nontrivial. We will show that this is impossible. Define $\Phi, \Psi: K \rightarrow \bar{c}\left(C(X)^{*}\right)$ by

$$
\begin{aligned}
\Phi(x) & =\operatorname{cl}\left\{\mu \in C\left(X^{*}\right): 0 \leqq \mu \leqq \sum_{i=1}^{n} \lambda_{i} T\left(x_{i}\right)^{+}, x\right. \\
& \left.=\sum_{i=1}^{n} \lambda_{\imath} x_{i}, \sum_{i=1}^{n} \lambda_{\imath}=1, \lambda_{i} \geqq 0\right\}
\end{aligned}
$$

and 


$$
\begin{aligned}
\Psi(x) & =\operatorname{cl}\left\{\mu \in C(X)^{*}: 0 \leqq \mu \leqq \sum_{i=1}^{n} \lambda_{i} T\left(x_{i}\right)^{-}, x\right. \\
& \left.=\sum_{i=1}^{n} \lambda_{i} x_{i}, \sum_{i=1}^{n} \lambda_{i}=1, \lambda_{i} \geqq 0\right\} .
\end{aligned}
$$

By Lemma 3.4, they are convex and lower semicontinuous. If we let $0 \neq \mu_{0} \in \Phi\left(x_{0}\right), \nu_{0} \in \Psi\left(x_{0}\right)$ such that $\left\|\mu_{0}\right\|=\left\|\nu_{0}\right\|$, Lemma 3.5 shows that we can find continuous selections $T_{1}, T_{2}$ of $\Phi, \Psi$ respectively, so that

$$
\left\|T_{1}(x)\right\|=\left\|T_{2}(x)\right\|, \quad x \in K
$$

and

$$
T_{1}\left(x_{0}\right)=\mu_{0}, T_{2}\left(x_{0}\right)=\nu_{0} .
$$

Note that for $x \in \partial K$,

$$
\begin{aligned}
& \Phi(x)=\left\{\mu: 0 \leqq \mu \leqq T(x)^{+}\right\}, \\
& \Psi(x)=\left\{\mu: 0 \leqq \mu \leqq T(x)^{-}\right\} .
\end{aligned}
$$

Hence if we let $T_{3}=T_{1}+T_{2}$, then $T_{3} \neq 0$ and for $x \in \partial K$

$$
\begin{aligned}
\left\|T(x)+T_{3}(x)\right\| & =\left\|T(x)^{+}+T_{1}(x)\right\|+\left\|T(x)^{-}-T_{2}(x)\right\| \\
& =\left\|T(x)^{+}\right\|+\left\|T_{1}(x)\right\|+\left\|T(x)^{-}\right\|-\left\|T_{2}(x)\right\| \\
& =\left\|T(x)^{+}\right\|+\left\|T(x)^{-}\right\|=1 .
\end{aligned}
$$

Similarly, we can show that $\left\|T(x)-T_{3}(x)\right\|=1$ for all $x \in \partial K$. This contradicts that $T$ is an extreme point of $S\left(A\left(K, C(X)^{*}\right)\right)$.

4. In this section, we will consider the set of extreme points of $S(L(C(X), A(K)))$ where $K$ is a simplex and $X$ is a metrizable compact Hausdorff space.

Theorem 4.1. Let $K$ be a simplex and let $X$ be a metrizable compact Hausdorff space. Then there is a one-to-one correspondence between the set of extreme points of $S(L(C(X), A(K)))$ and $C\left(\partial_{S} K\right.$, $X \cup-X)$ where $X \cup-X$ are contained in $C(X)^{*}$.

Proof. Let $T$ be an extreme point of $S(L(C(X), A(K)))=S(A(K$, $\left.C(X)^{*}\right)$ ). By Theorem 3.2, $T(\partial K) \subseteq X \cup-X$. We want to show that $T_{1}=T / \partial K$ is continuous with respect to the facial topology on $\partial K$. Let $E$ be a closed set in $X \cup-X$ and let

$$
E_{1}=E \cap X, E_{2}=E \cap(-X) .
$$

The set $\overline{\operatorname{conv}} E_{1}$ is a closed face of $S\left(C(X)^{*}\right)$ [1]. Consider the set $\partial K \cap T^{-1}\left(\overline{\mathrm{conv}} E_{1}\right)$, it is clear that 


$$
T_{1}^{-1}\left(E_{1}\right) \subseteq \partial K \cap T^{-1}\left(\overline{\operatorname{conv}} E_{1}\right)
$$

To prove the reverse inclusion, let $\left.x \in \partial K \cap T^{-1} \overline{(\operatorname{conv}} E_{1}\right)$. Then $T(x) \in$ $X \cap \overline{\operatorname{conv}}\left(E_{1}\right)$. Thus $T(x)$ is an extreme point of $\overline{\operatorname{conv}} E_{1}$ and $T(x) \in$ $E_{1}$, i.e., $x \in T^{-1}\left(E_{1}\right) \cap \partial K=T_{1}^{-1}\left(E_{1}\right)$. We conclude that

$$
T_{1}^{-1}\left(E_{1}\right)=\partial K \cap T^{-1}\left(\overline{\operatorname{conv}} E_{1}\right)
$$

and this shows that $T_{1}^{-1}\left(E_{1}\right)$ is facially closed (for $T^{-1}\left(\overline{\operatorname{conv}} E_{1}\right.$ ) is a closed face of $K)$. Similarly, $T^{-1}\left(E_{2}\right)$ is facially closed. Thus for each closed set $E \leqq X \cup-X, T_{1}^{-1}(E)$ is facially closed and hence $T_{1} \in C\left(\partial_{S} K\right.$, $X \cup-X)$.

Conversely, suppose $T_{1} \in C\left(\partial_{S} K, X \cup-X\right)$. Since every bounded subset of $C(X)^{*}$ is metrizable, by the remark of Theorem 2.2 we can extend $T_{1}$ to $T \in A\left(K, C(X)^{*}\right)$ and hence it is an extreme point of $S\left(A\left(K, C(X)^{*}\right)\right)$.

We call a simplex $K$ a Bauer simplex if and only if $\partial K$ is closed in $K$, or equivalently, $\partial_{S} K$ is Hausdorff. Recall also that on a Bauer simplex, the facial topology and the relative topology coincide and $A(K)$ is isometric to $C\left(\partial_{S} K\right)[1]$.

Proposition 4.2. (Morris-Phelps [7]). Let $X, Y$ be two compact Hausdorff spaces with $X$ metrizable. Then $S(L(C(X), C(Y)))$ is weak operator closed convex hull of its extreme points if and only if $Y$ is totally disconnected.

THeOREM 4.3. Let $K$ be a simplex and let $X$ be a metrizable compact Hausdorff space. Then the following are equivalent:

(i ) The unit ball $S(L(C(X), A(K)))$ is weak operator closed convex hull of its extreme points,

(ii) $\partial_{S} K$ is totally disconnected,

(iii) $A(K)$ is isometric to $C(Y)$ for some $Y$ which is compact Hausdorff and totally disconnected.

Proof. Note that if $\partial_{S} K$ is totally disconnected, it must be Hausdorff. Hence the equivalence of (ii) and (iii) follows from the above remarks on Bauer simplexes and that (iii) implies (i) follows from Proposition 4.2. To show that (i) implies (iii), by Proposition 4.2, we need only show that $K$ is a Bauer simplex, i.e., $\partial_{S} K$ is Hausdorff. To this end, for each $x \in \partial K$, we let $[x]=\{y \in \partial K$ : $¥$ facial neighborhoods $U, V$ of $x, y \in U \cap V=\varnothing\}$. Suppose $y \in[x]$. Then $f(y)=f(x)$ for all $f \in C\left(\partial_{S} K, X \cup-X\right)$ and by Theorem 4.1, $T(x)=T(y)$ for all extreme points $T \in S(L(C(X), A(K)))$. Since $S(L(C(X), A(K)))$ is the weak operator closed convex hull of its extreme points, we have 
$T(x)=T(y)$ for all $T \in S(L(C(X), A(K)))$. Suppose $x \neq y$. We let $R z$ be the subspace generated by a $z \in X$ and define $\varphi:[x, y] \rightarrow R z$ by $\varphi(\lambda x+(1-\lambda) y)=(1-\lambda) z$. Then $\varphi$ is a continuous affine function on the closed face $[x, y]$ of $K$. Hence there exists an extension $T_{\varphi} \in$ $A(K, R z) \subseteq A\left(K, C(Y)^{*}\right)$ and $T_{\varphi}(x) \neq T_{\varphi}(y)$. This leads to a contradiction. Thus $x=y$ and $\partial_{S} K$ is a Hausdorff space.

As a special case, we have:

Corollary 4.4. Let $K$ be a simplex. Then $A(K)$ is the closed convex hull of its extreme points if and only if $K$ is isometric to $C\left(\partial_{e} K\right)$ where $\partial_{e} K$ (the set of extreme points with the relative topology) is compact and totally disconnected.

The author would like to express his indebtedness to Professor R. R. Phelps for his valuable suggestions and careful reading of the manuscript.

\section{REFERENCES}

1. E. M. Alfsen, Compact Convex Sets and Boundary Integrals, Springer-Verlag, Berlin, 1971.

2. R. M. Blumenthal, J. Lindenstrauss, and R. R. Phelps, Extreme operators in $C(K)$, Pacific J. Math., 15 (1965), 747-756.

3. K. Lau, $A$ weaker condition on Michael's selection theorem, ditto notes.

4. A. J. Lazar, Affine functions on simplexes and extreme operators, Israel J. Math., 5 (1967), 31-43.

5. - Space of continuous functions on simplexes, Trans. Amer. Math. Soc., 134 (1968), 503-525.

6. E. Michael, Continuous selection I, Annals of Math., 63 (1956), 361-382.

7. P. D. Morris and R. R. Phelps, Theorems of Krein-Milman type for certain convex sets of operators, Trans. Amer. Math. Soc., 150 (1970), 183-200.

8. R. R. Phelps, Lectures on Choquet Theorem, Van Nostrand, Princeton, 1966.

9. M. Sharir, Characterization and properties of extreme operators into $C(Y)$, Israel J. Math., 12 (1972), 174-183.

Received February 10, 1973.

The University of Chicago 


\section{PACIFIC JOURNAL OF MATHEMATICS}

EDITORS

RICHARD ARENS (Managing Editor)

University of California

Los Angeles, California 90024

R. A. Beaumont

University of Washington

Seattle, Washington 98105

J. DugundJI

Department of Mathematics

University of Southern California

Los Angeles, California 90007

D. Gilbarg and J. Milgram

Stanford University

Stanford, California 94305

\section{ASSOCIATE EDITORS}

E. F. BECKENBACH

B. H. NeumanN

F. WOLF

K. YOSHIDA

\section{SUPPORTING INSTITUTIONS}

UNIVERSITY OF BRITISH COLUMBIA CALIFORNIA INSTITUTE OF TECHNOLOGY

UNIVERSITY OF CALIFORNIA

MONTANA STATE UNIVERSITY

UNIVERSITY OF NEVADA

NEW MEXICO STATE UNIVERSITY

OREGON STATE UNIVERSITY

UNIVERSITY OF OREGON

OSAKA UNIVERSITY

\author{
UNIVERSITY OF SOUTHERN CALIFORNIA \\ STANFORD UNIVERSITY \\ UNIVERSITY OF TOKYO \\ UNIVERSITY OF UTAH \\ WASHINGTON STATE UNIVERSITY \\ UNIVERSITY OF WASHINGTON \\ $\stackrel{*}{*} \stackrel{*}{*}{ }^{*}{ }^{*}{ }^{2}$ AMERICAN MATHEMATICAL SOCIETY \\ NAVAL WEAPONS CENTER
}




\section{Pacific Journal of Mathematics}

\section{Vol. 52, No. $1 \quad$ January, 1974}

David R. Adams, On the exceptional sets for spaces of potentials ............ 1

Philip Bacon, Axioms for the Čech cohomology of paracompacta ............ 7

Selwyn Ross Caradus, Perturbation theory for generalized Fredholm operators ..... 11

Kuang-Ho Chen, Phragmén-Lindelöf type theorems for a system of nonhomogeneous equations ............................ 17

Frederick Knowles Dashiell, Jr., Isomorphism problems for the Baire classes .......

M. G. Deshpande and V. K. Deshpande, Rings whose proper homomorphic images are right subdirectly irreducible . . . . . . . . . . . . . . . . . . . . . . . . .

Mary Rodriguez Embry, Self adjoint strictly cyclic operator algebras .............

Paul Erdős, On the distribution of numbers of the form $\sigma(n) / n$ and on some related

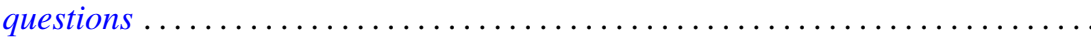

Richard Joseph Fleming and James E. Jamison, Hermitian and adjoint abelian

operators on certain Banach spaces ............................

Stanley P. Gudder and L. Haskins, The center of a poset .................. 85

Richard Howard Herman, Automorphism groups of operator algebras . . . ........

Worthen N. Hunsacker and Somashekhar Amrith Naimpally, Local compactness of families of continuous point-compact relations ....................

Donald Gordon James, On the normal subgroups of integral orthogonal groups ....

Eugene Carlyle Johnsen and Thomas Frederick Storer, Combinatorial structures in

loops. II. Commutative inverse property cyclic neofields of prime-power

order.

Ka-Sing Lau, Extreme operators on Choquet simplexes . . . . . . . . . . . . . . 129

Philip A. Leonard and Kenneth S. Williams, The septic character of 2, 3, 5 and $7 \ldots 143$

Dennis McGavran and Jingyal Pak, On the Nielsen number of a fiber map ........ 149

Stuart Edward Mills, Normed Köthe spaces as intermediate spaces of $L_{1}$ and

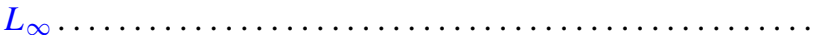

Philip Olin, Free products and elementary equivalence. .

Louis Jackson Ratliff, Jr., Locally quasi-unmixed Noetherian rings and ideals of the principal class.

Seiya Sasao, Homotopy types of spherical fibre spaces over spheres ...

Helga Schirmer, Fixed point sets of polyhedra ...

Kevin James Sharpe, Compatible topologies and continuous irreducible

representations.

Frank Siwiec, On defining a space by a weak base . . . . . . . . . . . . . . . 233

James McLean Sloss, Global reflection for a class of simple closed curves ....... 247

M. V. Subba Rao, On two congruences for primality . .

Raymond D. Terry, Oscillatory properties of a delay differential equation of even

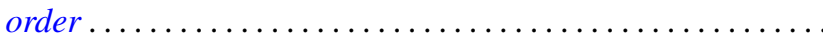

Joseph Dinneen Ward, Chebyshev centers in spaces of continuous functions . .

Robert Breckenridge Warfield, Jr., The uniqueness of elongations of Abelian

groups...

V. M. Warfield, Existence and adjoint theorems for linear stochastic differential

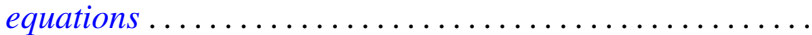

